\title{
MODALISATION AUTONYMIQUE ET DYNAMISME COMMUNICATIF
}

\author{
Gregor PERKO \\ Université de Ljubljana
}

\begin{abstract}
En): The aim of this article is to study the phenomenon of autonymic modalisation within the framework of communicative dynamism elaborated by Jan Firbas and his colleagues at the University of Brno. Special attention is given to the concept of "second instance". The autonymic modalisation, making dual use of a text item, involves two semiotic levels, objectlanguage level and metalinguistic level, and thus two parallel communicative dynamisms. The rhematisation at the metalinguistic level is the result of an ad hoc opposition between different metalinguistic aspects of an item depending heavily on different types of contexts.
\end{abstract}

Keywords (En): Functional Sentence Perspective; Communicative Dynamism, Semiotics, Metalinguistic Level, Autonymic Modalisation.

\section{Introduction}

Dans le présent article, nous nous proposons d'envisager la modalisation autonymique en termes de dynamisme communicatif. Nous essayerons de montrer que la modalisation autonymique, qui met en relief la «matérialité » du signe linguistique, ne présente nullement un dysfonctionnement de la communication qui provoquerait une rupture de la progression thématique, bien que «l'arrêt-surlangue » ou «l'arrêt-sur-énonciation » entraînent une sorte de «décrochage » communicatif. Plus précisément, l'énoncé se « dédouble » et se développe à deux niveaux distincts : un niveau mondain et un niveau métalinguistique.

Notre étude se limitera à la langue écrite et privilégiera les séquences modalisées explicites, accompagnées de commentaires métalinguistiques. Le corpus est constitué d'exemples tirés de la presse française et francophone (Le Monde, L'Express, Le Point, Jeune Afrique, Le Canard enchaîné) ou « glanés » sur la toile à l'aide du moteur de recherche Google.

\section{La modalisation autonymique}

La notion de modalisation autonymique ou réflexive, introduite par J. AuthierRevuz (AUTHIER-REVUZ, 1995), s'appuie sur la théorie sémiotique de J. ReyDebove et son concept de connotation autonymique. La connotation autonymique, qui fait partie de la dimension métalinguistique de la langue naturelle, désigne la situation «d'un signe qui signifie, comme connotateur, son signifiant et son signifié dénotatif »(REY-DEBOVE, 1997 : 253). La connotation autonymique se distingue de l'autonymie par le double emploi qu'elle fait du signe. Dans l'exemple (1), le signe politiquement correct, simple autonyme, ne renvoie pas au monde, mais uniquement à lui-même : il n'est pas en usage, il n'est qu'en mention.

(1) La locution politiquement correct est un calque de l'anglais politically correct. ${ }^{1}$

\footnotetext{
${ }^{1}$ On ne peut qu'être d'accord avec J. Authier-Revuz (AutHIER-RevuZ, $2003: 77$ ) qui voit, en dehors de quelques rares cas d'ambiguité « irréductible», dans les signes typographiques qui accompagnent
} 
Il n'en est pas de même dans (2), où, sous l'effet de l'emploi des guillemets et de la glose métalinguistique (comme on dit aujourd'hui), ce même signe est à la fois en usage (il réfère au monde) et en mention (il se signifie lui-même).

(2) Le pharisien ne fut pas inspiré ce jour-là, et le comportement de Jésus ne fut pas, comme on dit aujourd'hui, «politiquement correct ». (http://evangeli.net. Consulté le 20/5/2011)

Comme le montre (3), forgé à partir l'exemple précédent, la glose n'est pas toujours indispensable pour signaler ce double usage: des signes typographiques, en l'occurrence les guillements, suffisent (voir plus loin) :

(3) Le pharisien ne fut pas inspiré ce jour-là, et le comportement de Jésus ne fut pas «politiquement correct ».

L'adoption d'une perspective énonciative conduit J. Authier-Revuz à proposer une notion moins «statique » que celle de connotation autonymique limitée à la structure sémiotique du signe linguistique. La modalisation autonymique, qui permet à l'énonciateur de commenter son dire «en train de se faire », constitue une configuration hétérogène et opacifiante, une énonciation «dédoublée » cumulant deux sémiotiques : l'usage d'un segment pour dénoter une chose (usage standard du signe) et le retour en mention de ce même segment (usage autonyme du signe).

À l'oral, la modalisation autonymique est signalée par l'intonation (voir la note 8 plus loin et surtout POTTS, 2004, 2007), celle-ci étant parfois accompagnée par la gestuelle (par exemple, par le «geste du guillemet»). À l'écrit, ce sont le plus souvent les guillemets, les italiques et parfois les gras qui marquent l'autonymisation d'un segment textuel :

(4) D'ordinaire, dans les familles, c'est plutôt le père qui veille à la « respectabilité » des enfants. Chez les Le Pen, c'est plus que le contraire : c'est la fille qui doit contrôler les débordements du père. (Le Canard enchaîné du 3/8/2011)

Sans les guillemets, la mise en cause de la pertinence de l'emploi de respectabilité dans le contexte donné passerait inaperçue aux yeux du lecteur. $\mathrm{Vu}$ la polysémie de ces moyens typographiques, les guillemets, les italiques et les gras seuls ne suffisent pas toujours à signaler la séquence modalisée :

(5) Les députés sont «furax », paraît-il. Ça aurait même « gueulé » dans les couloirs de l'Assemblée, témoigne un pilier de la buvette au Palais-Bourbon. (Le Canard enchaîné du $3 / 8 / 2011)^{2}$

les autonymes (guillemets, italiques) un phénomène de «surmarquage», dû à une pression normative quasi-fétichiste des logiciens : les énoncés comportant des signes autonymes ne sont pas prononcés avec une courbe différente de celle des énoncés « analogues » en usage (Le mot chou n'est pas invariable. vs Le gros chou est immangeable.).

${ }^{2}$ Les gras sont dans le texte. 
Dans l'exemple (5), les guillemets pourraient ne signaler qu'une citation. Les caractères gras apportent un marquage complémentaire qui signale que les deux mots doivent être interprétés dans leur dimension autonymique: les mots employés par le parlementaire relèvent du registre familier et traduisent nettement de l'agacement.

Le marquage des séquences modalisées s'accompagne souvent de commentaires métalinguistiques qui explicitent l'aspect autonymique de la séquence modalisée ${ }^{3}$. En voici deux exemples (nous soulignons) :

(6) [Ce nationaliste] participa à ce titre à l'attaque d'une gendarmerie, à Pietrosella (Corsedu-Sud), où a été dérobé le pistolet ayant servi pour l'assassinat. Mais, quand il s'est agi d'augmenter le niveau de violence, c'est-à-dire d'abattre, au sens propre, « un symbole » de l'État français, Versini fut apparemment le seul à refuser de participer au meurtre. (L'Express du 26/5/2011)

(7) Je lui fais remarquer que, avec ou sans talonnettes, on se grandit toujours à sortir avec un top model. Et je note ce glissement de vocabulaire: les idiots sortent avec des «mannequins », les malins avec des «top model». («Le journal de Carla B. », Le Canard enchaîné du 27/7/2011)

La modalisation autonomique est une des manifestations de l'hétérogénéité inhérente à la langue et à l'énonciation ${ }^{4}$. Dans les exemples (4), (6) et (7), la modalisation repose sur une hétérogénéité sémantique du mot à l'intérieur de la langue elle-même. Dans (5), on observe l'irruption, dans l'énonciation du journaliste, du discours d'un autre, d'un « discours autre ».

La typologie établie par J. Authier-Revuz pour cerner cette hétérogénéité repose sur différentes formes de non-coïncidence, réelle ou supposée, entre les éléments intervenant dans l'acte de l'énonciation. Elle distingue schématiquement entre :

- une non-coïncidence à l'intérieur de l'interlocution entre les points de vue du locuteur et de l'allocutaire (AUTHIER-REVUZ, 1995 : 163-234) :

(8) «J'espère que ça sera fait dans de bonnes conditions et que le scrutin sera régulier, si vous voyez ce que je veux dire », a lancé Ségolène Royal [...]. (Le Point du 11/01/2011)

- une non-coïncidence du discours à lui-même (AUTHIER-REVUZ, 1995 : 235505) :

(9) Voilà, avec le débat voulu par l'Elysée et l'UMP sur la laïcité et l'islam, une controverse où le calcul et la posture - voire l' «hystérie » pour reprendre le mot de Jean-François Copé l'emportent sur la pédagogie et l'intérêt commun. (Le Monde du 1/4/2011)

Ce type de modalisation autonymique fait partie d'un ensemble plus large, celui de la représentation du «discours autre » incluant notamment le discours

\footnotetext{
${ }^{3}$ Pour un panorama de diverses formes qu'adoptent les commentaires métalinguistiques, voir AUTHIER-REvUZ (1995: 101-160).

${ }^{4}$ Parmi les assises théoriques sur lesquelles repose l'approche de J. Authier-Revuz, citons l'approche discursive de Michel Pêcheux, le dialogisme de Mikhail Bakhtine, le concept d'intersubjectivité d'Émile Benveniste et la notion de «sujet clivé » de Jacques Lacan.
} 
rapporté, mais ne se confondant avec aucune forme de celui-ci, étant la seule à cumuler les deux modes sémiotiques : l'usage et la mention ${ }^{5}$.

- une non-coïncidence entre le mot et la chose/le référent (AUTHIER-REVUZ, 1995 : 507-712) :

(10) Le présent paragraphe a pour objet de présenter les « catas » [est-ce le mot juste ?] liées au domaine militaire qui ont eu un impact écologique, mais aussi humain (http://fr.wikipedia.org. Consulté le 20/5/2011)

Cette non-coïncidence, qui repose sur le caractère arbitraire du signe linguistique, va à l'encontre du sentiment du sujet parlant qui tend à identifier instinctivement la langue et le monde/le réel.

- non-coïncidence du mot à lui-même (AUTHIER-REVUZ, 1995 : 713-801) :

(11) La Hijra a constitué une épreuve à tous points de vue : ce n'est qu'après une longue traversée du désert, dans le sens propre et figuré du terme, que les premiers croyants ont pu enfin vivre leur foi en toute quiétude : l'entrée victorieuse du Prophète à La Mecque en 630 constitue une étape cruciale dans l'évolution de l'islam. En effet, les musulmans passaient d'un régime d'oppression à une situation plus sécurisante. (http://ouma.com. Consulté le 20/5/2011)

Le commentaire métalinguistique «dans le sens propre et figuré du terme » marque que l'élément «une longue traversée $d u$ désert» est équivoque, susceptible d'une double interprétation, ce qui «met en question » l'unité du signe linguistique.

\section{Le dynamisme communicatif}

Le phénomène de la modalisation (ou de la connotation) autonymique a été essentiellement abordé dans ses dimensions sémiotique, syntaxique et, plus tard, énonciative mettant en évidence différents effets de discours. À notre connaissance, le phénomène n'a jamais été analysé de façon systématique dans une perspective textuelle. L'étude que nous proposerons s'appuiera sur le concept de «dynamisme communicatif» élaboré par Jan Firbas et ses collègues de l'Université de Brno (FIRBAS, 1971, 1974, 1992). L'avantage marquant de ce modèle réside justement dans son caractère «dynamique », souple, qui permet de dépasser la rigidité de certaines approches reposant sur des dichotomies statiques (connu / non-connu ou information donnée / information nouvelle).

Le dynamisme communicatif désigne une propriété fondamentale de la communication qui se manifeste dans le développement de l'information qu'on veut transmettre et qui consiste à faire avancer ce développement. Différents éléments de l'énoncé contribuent, à des degrés différents, à ce développement : plus un élément fait avancer la communication, plus son degré de dynamisme est élevé. Ces éléments forment un continuum délimité, d'un côté, par le rhème

\footnotetext{
${ }^{5}$ La position de J. Authier-Revuz qui traite le discours direct comme un cas d'autonymie «simple » ou comme une forme discursive qui fait « usage de la mention » (AUTHIER-REVUZ, 2003: 84) est loin de faire l'unanimité et a donné lieu à une importante polémique (cf. RosiER, 1999, 2008 ; KOMUR-THILLOY, 2010).
} 
propre, portant le degré de dynamisme le plus élevé, et de l'autre, par le thème propre, dépendant largement du contexte précédent et portant le degré de dynamisme le plus bas. Entre ces deux pôles, Firbas distingue plusieurs types d'éléments qui connaissent divers degrés de dynamisme communicatif: le diathème, le reste du thème, le reste du diathème, la transition propre, l'élément axé sur la transition, le reste de la transition, le reste du rhème, etc. :

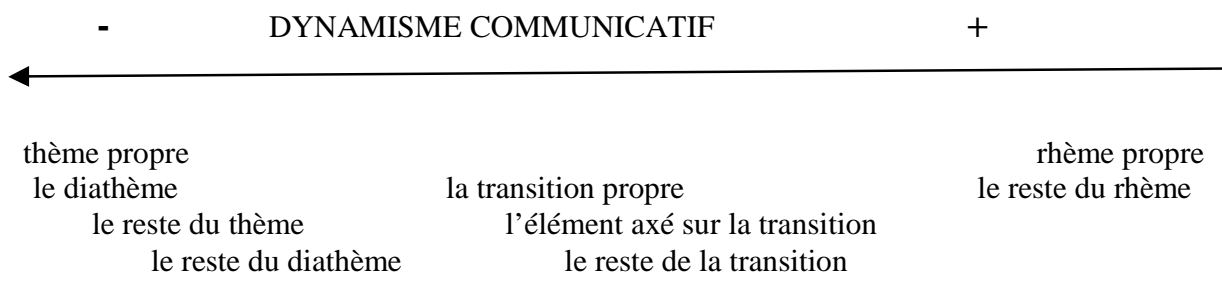

L'attribution des degrés dépend de l'interaction entre trois facteurs: le contexte, la structure sémantique et l'arrangement linéaire de l'énoncé, auxquels on peut ajouter le rôle important joué par le dispositif prosodique 6 . Bien que ces facteurs puissent souvent agir dans le même sens, le dynamisme communicatif ne doit pas être réduit à l'action d'un seul facteur. Un thème, par exemple, qui d'ailleurs n'est pas obligatoirement présent dans tout énoncé, n'est pas toujours contextuellement dépendant, il n'est pas nécessairement placé au début de l'énoncé, n'occupe pas toujours la position du sujet et ne se confond pas forcément avec « ce dont on parle».

Une attention particulière est accordée à l'interaction entre les énoncés constituant un texte. Firbas distingue entre deux niveaux : un niveau de première instance et un niveau de seconde instance. Le premier regroupe des énoncés qui sont soit entièrement (niveau de base, ang. basic instance level) soit partiellement (niveau ordinaire, ang. ordinary instance level) indépendants du contexte. Le second (ang. second instance level) ${ }^{7}$, qui nous intéressera davantage par la suite, regroupe des énoncés dont la dépendance contextuelle est maximale, mais dont l'un des éléments non rhématiques est mis en contraste et, en conséquence, rhématisé. Dans les exemples qu'en donne Firbas (FIRBAS, 1968 : 12-18; 1992 : 110-114), ces rhématisations touchent principalement les éléments de transition, et notamment les valeurs temporelles, aspectuelles ou modales des verbes: un énoncé comme John WENT home peut s'opposer implicitement à John DID GO

\footnotetext{
${ }^{6}$ La prosodie ne peut pas être traitée au même niveau d'analyse que les autres facteurs cités, étant donné que la prosodie n'est, en principe, que le «reflet» de l'interaction entre les facteurs et ne constitue donc pas un facteur «indépendant». Cependant, vu l'intérêt que porte J. Firbas au dispositif prosodique, traduit à l'écrit par des conventions typographiques (les capitales, les gras, la taille de caractères etc.), on est tenté de le traiter sur le pied d'égalité avec le contexte, la sémantique et l'ordre des mots.

${ }^{7}$ Le concept de «seconde instance » est emprunté à Dwight L. Bolinger (BOLINGER, $1952: 1123$ ) et désigne des phénomènes qui vont à l'encontre de la tendance générale de l'arrangenent linéaire de l'énoncé vers une valeur communicative toujours plus grande: "gradation of position creates gradation of meaning when there are no interfering factors » (BOLINGER, 1952:1125). Ce concept est à rapprocher du concept d'emphase, mieux connu en linguistique contemporaine.
} 
home, John DIDN'T GO home, John WILL GO home, John CAN GO home etc. Dans un contexte particulier et pour dissiper une ambiguïté, un malentendu ou faire une rectification, un énoncé qui mettrait en relief la valeur temporelle et assertive de did go dans John DID GO home serait un énoncé tout à fait pertinent et où l'élément did go porterait le degré le plus haut de dynamisme communicatif.

\section{La modalisation autonymique et le dédoublement du dynamisme communicatif}

L'étude du fonctionnement textuel de l'autonymie «simple» ne semble poser, au moins pour ce qui nous intéresse, aucun problème particulier, vu qu'elle n'implique qu'un seul niveau sémiotique :

(12) Courir est un verbe de mouvement, mais à la différence de marcher, il peut être suivi d'un complément de destination.

(http://ethiopiques.refer.sn/spip.php?article742. Consulté le 12/9/2011)

L'élément courir qui présente le point de départ de l'énoncé se base sur le contexte précédent apportant une analyse contrastive de verbes de mouvement ou de déplacement en anglais, français et wolof. L'élément porte un degré de dynamisme communicatif très bas et constitue par conséquent le thème propre de l'énoncé auquel se rapporte le commentaire métalinguistique «il peut être suivi d'un complément de destination» présentant la partie non thématique. L'élément « mais à la différence de marcher » a la fonction de diathème (voir FIRBAS, 1992 : 79-81).

La situation est nettement plus complexe lorsque l'on a affaire à la modalisation autonymique étant donné qu'elle implique deux niveaux sémiotiques. Il semble légitime de postuler que les énoncés modalisés connaissent deux dynamismes communicatifs indépendants l'un de l'autre.

Les travaux de J. Firbas et de ses collègues ne s'intéressaient qu'au niveau sémiotique en usage. Le premier, à notre connaissance, qui ait fait un rapprochement explicite entre la «seconde instance» et la modalisation autonymique, a été Bernard COMBETTES (1983: 21). Voici l'exemple qu'il donne pour illustrer ce qu'il appelle la «métacommunication» :

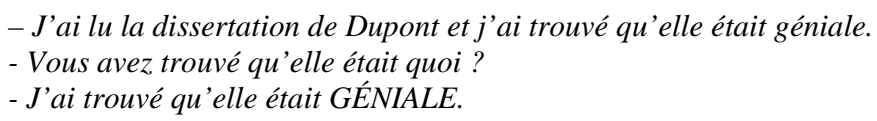

- J'ai lu la dissertation de Dupont et j'ai trouvé qu'elle était géniale.

- Vous avez trouvé qu'elle était quoi?

- J'ai trouvé qu'elle était GÉNIALE.

La rhématisation de l'adjectif génial est marquée par l'accent focal qui est signalé à l'écrit par les majuscules. Cet accent opère typiquement au niveau sémiotique en usage. En répétant plus ou moins littéralement ce qu'il a dit, mais avec une prosodie différente, le locuteur fait clairement savoir qu'il le pense vraiment et qu'il ne changera pas d'avis. B. Combettes (COMBETTES, 1983: 21) ajoute que cette accentuation peut s'accompagner d'un «détachement des 
syllabes », GE-NIA-LE, ce qui est le marquage typique des éléments en mention ${ }^{8}$. Ce détachement, en «détournant» l'attention sur la matérialité du signe linguistique ${ }^{9}$, signale une non-coïncidence de points de vue entre le locuteur et l'allocutaire.

Notre étude reprend maintenant la notion de «seconde instance » que nous venons de présenter brièvement. Comme nous avons vu plus haut, cette notion repose sur deux conditions nécessaires :

- $\quad$ l'élément doit connaître une forte dépendance contextuelle,

- cet élément doit être mis en contraste, ce qui entraîne sa rhématisation.

Pour les besoins de notre analyse, nous employons le terme de «contexte» dans son acception «large » que l'on trouve, par exemple, dans la grammaire cognitive de Ronald W. Langacker qui distingue entre trois types de contextes (LANGACKER, 1987 : 401-408) :

- $\quad$ contexte systémique : correspondant au système linguistique ;

- contexte situationnel : englobant les facteurs extralinguistiques et la dimension pragmatique ;

- contexte syntagmatique (ou cotexte): se rapportant aux combinaisons dans lesquelles entre une unité linguistique.

Revenons à l'exemple (13) qui satisfait aux deux conditions citées plus haut : l'énoncé final s'appuie sur le contexte syntagmatique (le locuteur répète plus ou moins littéralement ce qu'il a dit) et révèle un contraste, une non-coïncidence entre les avis des deux interlocuteurs concernant l'acception du mot génial et la pertinence de l'emploi de celui-ci dans le contexte donné.

Le clivage entre les deux niveaux sémiotiques qui, dans (13), n'a été signalé que par la possibilité d'appliquer deux dispositifs prosodiques différents, traduits, à l'écrit, tant bien que mal, par des moyens typographiques (les majuscules et les tirets), peut être marqué explicitement par la présence d'un commentaire métalinguistique. Prenons l'exemple suivant, trouvé sur un forum internet, dans la rubrique où l'on discute des «performances des courses de voile ». À un moment de la discussion, Kero Zen s'en prend plus directement à un des participants (appelé «Chico678»). En voici un extrait :

(14) Ah ! Chico, mon ami Chico. Je suis toujours un peu surpris lorsque je vois des gens d'une intelligence supérieure se laisser aller à des petits calculs mesquins, et utiliser des excuses d'une telle platitude qui me laissent entrevoir les faiblesses congénitales qu'une éducation policée n'a pas réussi à éradiquer entièrement.

C'est donc avec une sorte de compassion que je me vois contraint de t'indiquer que si tu lambinais avec quelques autres lambins, c'est que tu n'avais pas fait les bons choix stratégiques,

\footnotetext{
${ }^{8}$ Pour Ch. Potts (POTTS, 2004 : 136-139), qui distingue nettement entre l'accent focal et l'accent « de citation ", la prosodie de l'élément en mention se caractérise par un détachement des unités prosodiques qui adoptent chacune un contour montant-descendant-montant. : "In quotation, each prosodic word projects to its own intonational phrase with a rise-fall-rise contour. » (POTTS, 2004 : 137)

${ }^{9}$ Peut-être pourrait-on parler d'un phénomène d'iconicité linguistique : en prolongeant la durée de la prononciation du signe, on « amplifie » le signifiant et, en même temps, tout le signe.
} 
que ta situation, certes désagréable n'en était pas moins conforme au raisonnement qui avait prévalu dans ta décision. A contrario, si je paradais, pour employer le terme que tu utilises (a), bien qu'il ne corresponde pas tout à fait à ma modestie naturelle (b), c'est que les choix retenus ont été judicieux.

Et, comme tu le reconnais un peu plus loin, avec beaucoup d'à propos, une prévision météo fantaisiste pouvant coincer un navigateur dans une pétole importante, j'en ai été victime et donc retenu contre mon gré dans une zone que je ne demandais qu'à quitter.

(http:// sport.francetv.fr. Consulté le 12/9/2011)

La modalisation autonymique du verbe parader est signalée par deux commentaires: pour employer le terme que tu utilises (a) et bien qu'il ne corresponde pas à ma modestie naturelle (b). Le premier commentaire (a) qui s'appuie sur le contexte syntagmatique de gauche révèle un contraste, une noncoïncidence entre les coénonciateurs, le second (b), reposant sur un contexte situationnel plus vaste mobilisant des connaissances encyclopédiques, insiste sur une inadéquation du mot à la réalité. Cette double mise en contraste et par conséquent la rhématisation de parader ne concerne cependant que le niveau en mention. Le degré de dynamisme communicatif de cet élément est tout autre si l'on s'en tient au niveau en usage où cette séquence se place dans la partie thématique contribuant peu au développement de la communication.

Dans certains cas de modalisation autonymique reposant sur une noncoïncidence interdiscursive, on peut avoir affaire à un contexte syntagmatique non immédiat, voire implicite, qu'il est parfois difficile de distinguer du contexte situationnel :

(15) Pour les opérateurs économiques, la mesure obéit au principe de réalité, qui exige de mettre l'Algérie au diapason de l'économie occidentale. Enfin presque, puisque le nouveau week-end algérien est «semi-universel », selon l'expression consacrée en Algérie.

(Jeune Afrique du 11/8/09)

Dans cet exemple, à la différence du précédent, la seconde instance rhématise, au niveau en mention, l'élément qui constitue le rhème également au niveau en usage.

Le contexte situationnel peut faire intervenir des considérations d'ordre pragmatique ou socioculturel, qui mènent à des cas de non-coïncidence dans l'interlocution :

(16) La conclusion que je livre à la lumière de la politique suivie par Papadopoulos depuis son arrivée au pouvoir est la suivante : la raison principale de l'impasse dans laquelle se trouve Chypre aujourd'hui réside dans le fait que les élites grecques de l'île ne sont pas prêtes à partager le pouvoir avec les élites turques. Quant aux autres raisons du blocage, soit elles découlent de celle-ci soit elles viennent la renforcer. De ce point de vue, les paroles de Papadopoulos diffusées dans l'Express du 5 mai dernier ont valeur d'aveu. Le leader chypriote, si l'on me pardonne l'expression, crache le morceau.

(http://turquieeuropeenne.eu/spip.php?page=print\&id_article=1287. Consulté le 20/5/2011)

Dans l'exemple suivant (17), la modalisation, mettant en contraste des aspects sémantiques du signe linguistique (remotivation étymologique du nom propre), prend appui sur le contexte systémique : 
(17) Dans Potiche, mon personnage est sur le fil, entre le réel et la caricature. L'hôtesse de l'air que je joue dans Low Cost est plus nuancée, c'est le cas de le dire, puisqu'elle s'appelle Nuance... ( « Judith Godrèche en quatre vérités », L'Express du 8/6/11)

\section{Conclusion}

La modalisation autonymique permet de faire double usage d'une séquence textuelle, à deux niveaux sémiotiques différents : au niveau mondain et au niveau métalinguistique. Cette séquence s'inscrit dans deux dynamismes communicatifs indépendants l'un de l'autre. La rhématisation de la séquence modalisée au niveau en mention repose sur le principe de la «seconde instance » : la mise en contraste d'un aspect métalinguistique de cette séquence caractérisée par un degré élevé de dépendance contextuelle. Nous devons distinguer plusieurs types de contrastes (ou de non-coïncidences) qui s'appuient sur différents types de contextes :

- le contraste portant sur les participants d'une interlocution et s'appuyant essentiellement sur le contexte situationnel ;

- le contraste portant sur le discours et s'appuyant sur le contexte syntagmatique immédiat ou éloigné, voire implicite ;

- $\quad$ le contraste portant sur la dénomination, c'est-à-dire sur la relation entre le mot et son référent, et s'appuyant essentiellement sur le contexte situationnel ;

- $\quad$ le contraste portant sur le signe lui-même et s'appuyant sur le contexte systémique.

\section{BIBLIOGRAPHIE}

BOLINGER Dwight L. (1952), Linear modification, Publications of the Modern Language Association of America 67, p. 1117-1144.

AUTHIER-REVUZ Jacqueline (1995), Ces mots qui ne vont pas de soi, Paris, Larousse.

AUTHIER-REVUZ Jacqueline (2003), Le fait autonymique: langage, langue discours - quelques repères, in: AUTHIER-REVUZ Jacqueline et al. (éds.), Parler des mots : Le fait autonymique en discours, Paris, Presses Sorbonne Nouvelle, p. 67-97.

COMBETTES Bernard (1983), Pour une grammaire textuelle: la progression thématique, Bruxelles, De Boeck-Duculot.

FIRBAS Jan (1968), On the prosodic features of the modern English finite verbs as means of functional sentence perspective, Brno Studies in English 7, p. 11-48.

FIRBAS Jan (1971), On the concept of communicative dynamism in the theory of functional sentence perspective, Sborník prací Filosofické fakulty brněnské university A 19, pp. 135-144.

FIRBAS Jan (1974), Some aspects of the Czechoslovak approach to problems of functional sentence perspective, in: DANEŠ František (éd.), Papers on Functional Sentence Perspective, Prague/La Haye/Paris, Academia/Mouton, p. 11-37.

FIRBAS Jan (1992), Functional Sentence Perspective in Written and Spoken Communication, Cambridge, Cambridge University Press. 
Komur-Thilloy, Greta (2010), Presse écrite et discours rapporté, Paris, Orizons.

LANGACKER Ronald W. (1987), Foundations of Cognitive Grammar, volume 1 (Theoretical Prerequisists), Stanford, Stanford University Press.

POTTS Christopher (2005), Lexicalized intonational meaning, in KAWAHARA Shigeto (éd.), University of Massachusetts Occasional Papers 30, Amherst, GLSA, pp. 129-146.

POTTS Christopher (2007), The dimensions of quotation, in : BARKER Chris, JACOBSON Pauline (éds.), Direct Compositionality, Oxford, Oxford University Press, p. 405-431.

REY-DeBOVE Josette ( ${ }^{2} 1997$ [1978]), Le métalangage, Paris, Armand Colin.

ROSIER Laurence (1999), Le discours rapporté: histroire, théories, pratiques, Bruxelles, De Boeck-Duculot.

ROSIER Laurence (2008), Le discours rapporté en français, Paris, Ophrys. 